\title{
Integrated digitization method for cultural heritage objects
}

\author{
Grzegorz Mączkowski, ${ }^{1}$ Robert Sitnik, ${ }^{1}$ and Jakub Krzesłowski ${ }^{1}$ \\ ${ }^{\text {I}}$ Faculty of Mechatronics, Warsaw University of Technology, Boboli 8, 02-525 Warsaw, Poland
}

Received May 11, 2012; accepted June 15, 2012; published June 30, 2012

\begin{abstract}
Digitization of cultural heritage objects recently becomes a very promising tool for storing information about precious artifacts. This work shows an integrated, multimodal measurement method for digitization of cultural heritage objects with the example of a representative metal figurine. We propose an optical measurement system which uses structure light projection, multispectral color acquisition and Bi-Directional Reflectance Distribution Function (BRDF) estimation. Accordingly, an implementation of data processing path is explained with an illustration of its main steps and the final result.
\end{abstract}

The proposed method aims to capture three different features characteristic for the digitized object: its shape, color and reflectance properties. For illustration we present its implementation with a custom measurement system which was used to digitize a metal figurine of the $215 \times 95 \times 95 \mathrm{~mm}$ size, originated from China, which depicts the reproduction of a soldier from the Chinese terracotta army (Fig. 1) [1]. It is made of casted metal painted to resemble patinated bronze. It was chosen as representative for presentation of the proposed technology because the surface has large variations of reflection properties and color and has a complicated shape.

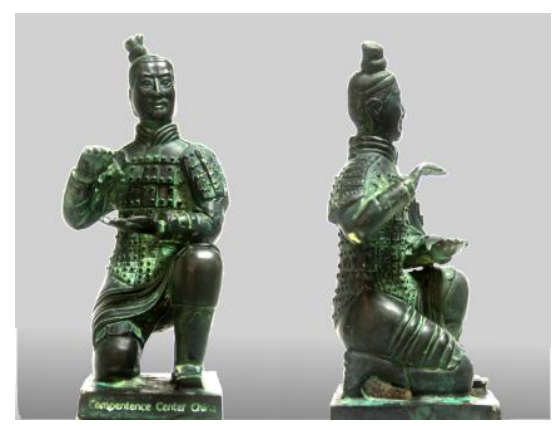

Fig. 1. Test object used for digitization.

The shape information is represented as a cloud of points in three-dimensional space. There are several methods of registering the shape this way, such as laser scanning [2], structured light projection [3], shape from silhuette [4], etc. In our work we focus mainly on the development of techniques employing structured light projection, because they offer very accurate results, they are scalable and do not require expensive equipment [5]. The shape measurement system comprises a digital camera (charged coupled device) and a digital light projector which projects sine fringe patterns with a variable phase. The temporal phase shifting [6] method is used to retrieve shape information from registered images. Additionally, the camera is geometrically calibrated so that the influence of its aberrations is reduced [5]. The device acquires clouds of points with an average resolution of 100 points per square millimeter. However, it can be increased by modifying the camera lens and measurement volume. Figure $2 \mathrm{a}$ shows the concept of the shape measurement system along with its working range where the scanned object is placed.

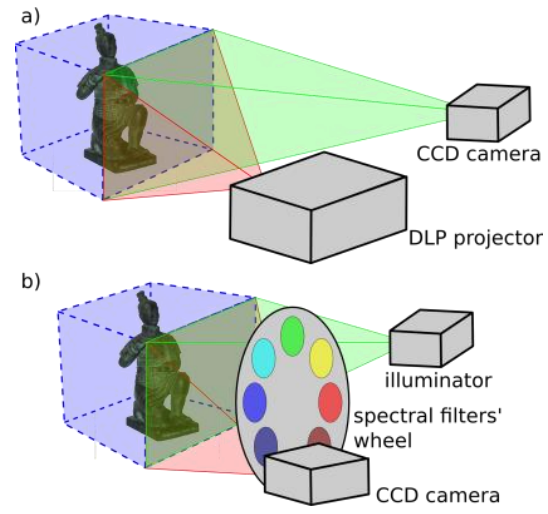

Fig. 2. Outline of setup: a) for shape measurement; b) for color measurement.

Some attempts to capture color information utilize off the shelf color cameras which register three color bands: red, green and blue. Such information is insufficient for spectral reflectance reconstruction and mapping to CIE XYZ color coordinates can only be made. Normally, color camera response is dependent on the illumination spectrum and spectral sensitivity of the sensor. There are many different solutions which address these limitations. Some of them rely on proper calibration of the camera [78], whereas others use an increased number of spectral channels used for acquisition [9] which gives very accurate results, especially for flat surfaces where illumination direction is well defined [10-11].

In our setup color information is registered with the use of a custom built multispectral camera with 10 bands within the visible spectrum. Its filtering is performed by a set of interference filters which are placed in front of the detector and can be automatically switched. The measurement is performed in strictly controlled illumination conditions in a dark room, so that only a 
dedicated halogen light source is used [12]. The measurement system interpolates spectral reflectance based on spectral channels output and calculates color in the XYZ color space which can be further processed for display. Figure $2 \mathrm{~b}$ shows an outline of the multispectral color registration system.

The third property to register during digitization is the angular reflectance distribution function [13]. Since it addresses a complicated physical phenomenon there are several mathematical models that simplify its description [14-15]. Usually their parameters are calculated from a set of images registered with different illumination and observation directions [16]. In the presented setup we use a grid of illuminators which uniformly emit light from several directions [17]. The measurement data serve for calculating coefficients for the Phong model, which is widely used in computer graphics [18].

The significant feature of the presented system is the use of a single detector for all three measurement stages. This allows for explicit data alignment. Every point on the surface, which is registered by a single detector pixel has a determined spectral and angular reflectance. This fact simplifies also further data processing stages. The final look of the integrated measurement system is presented in Fig.3.

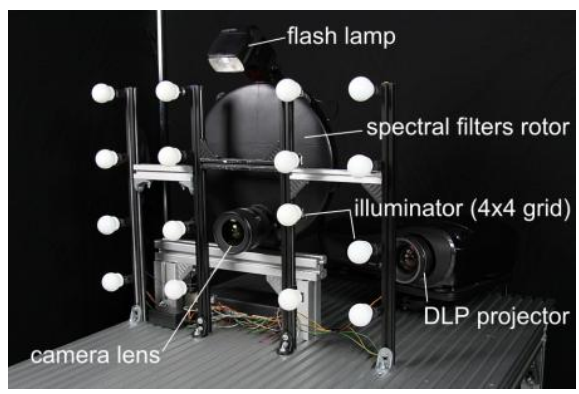

Fig. 3. Complete setup ready for measurement.

Data processing is performed with the use of 3DMADMAC (3D Measurement with Algorithms for Directional Merging And Conversion) software system [19]. It uses a set of plugins which implement basic operations on volumetric data and can be combined in complex calculation patterns. This way all computing stages are performed in a single environment and can be automated for efficient processing of big data sets.

Raw data registered during measurement consist of sets of images captured by the camera which need to be processed to extract information about significant features of the object. The data processing path is divided into two main stages (Fig. 4). The first one is conducted directly after the measurement and serves to prepare a digital model with the highest precision for storage purposes. It outputs directional clouds of points in response to spectral reflectance and light power according to illumination and observation directions. At this stage it is inconvenient to display or interpret the data, so the second calculation step is introduced to prepare of the final result. It is further divided into two phases:

1. with operations independent for all measured object properties and;

2. merging stage when the shape is used to refine spectral and directional reflectance

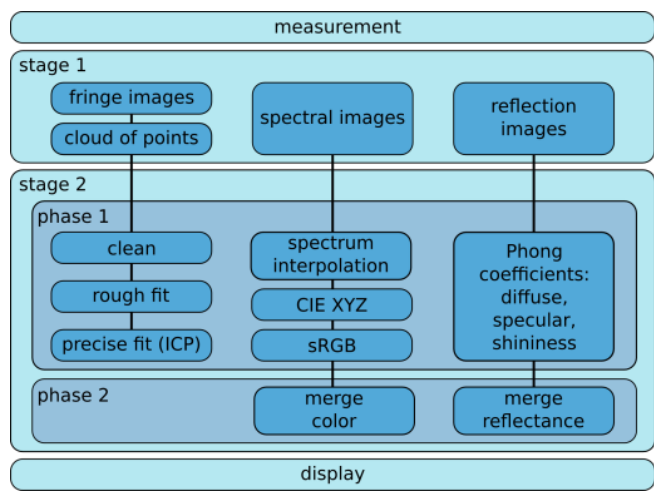

Fig. 4. Diagram illustrating data processing path.

Phase one serves to calculate the XYZ and RGB color space from spectral data which is a straightforward operation [20], to calculate the BRDF Phong model coefficients described previously [21] and merge directional clouds of points [22]. The single measurement procedure registers the object from one direction. In order to obtain a complete model of the surface it is necessary to scan it from several directions and then merge the data by fitting single clouds to each other, changing their transformations in the coordinate system. Initially, the clouds are fitted roughly, manually or semi-automatically using similar topological features existing in different clouds. Eventually, the clouds are fitted precisely with the aid of ICP (Iterative Closest Points) procedures [23].

Consequently, phase one results in a complete shape model, RGB texture information and Phong parameters of directional reflectance. Unfortunately, different registration directions lead to texture non-uniformity due to illumination variations. Therefore a second phase of calculation process is necessary [24-25]. Because the geometry of the measurement system is fixed, so that the position of the detector and the light source does not change between measurements, the only variable is the local surface orientation represented as a vector normal to the surface in each registered point. The angle between the observation direction and the normal vector is used as a weight for averaging color coordinates from overlapping clouds of points in the small neighbourhood of a currently analyzed point. The points which are registered opposite to the detector are favoured over the ones which are visible from the small angle (Fig. 5). The result of texture refining is visible in Fig. 6. Similar operation is performed for BRDF diffuse, specular and shininess coefficients. 


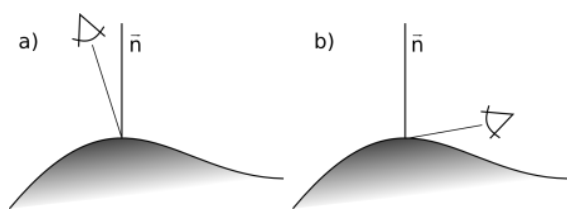

Fig. 5. Observation direction during measurement relative to surface orientation: a) favoured case; b) insignificant case

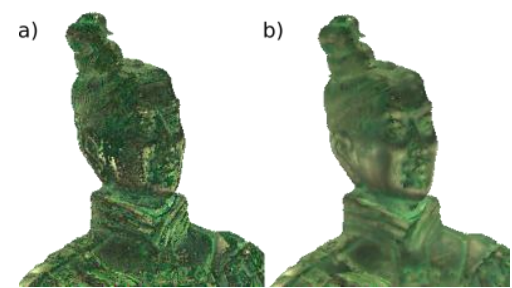

Fig. 6. Result of color merging algorithm: a) before operation; b) after operation

In the end, the final result of data processing can be displayed in dedicated visualization software which is capable of interpreting the directional reflectance information in real time. The user can change the direction of observation and the direction of illumination to observe differences in object reflectance. Some examples are shown in Fig. 7. The final digitized model is a cloud containing approximately 15 million points. The average distance between them is approximately $0.1 \mathrm{~mm}$, which corresponds to the resolution of the system. The accuracy of the measurement can be assessed with the use of a known reference object, as described in work [5]. In this case, the RMS (Root Mean Square) error of plane fitting to the planar reference object is $0.078 \mathrm{~mm}$ for $99.7 \%$ of points, corresponding to three times the standard deviation of the plane fit error.

Every point has assigned color coordinates in sRGB [26] color space and three BRDF coefficients conforming to the Phong model. The color coordinates are calculated from spectral response, established with $0.032 \%$ mean relative RMS, comparing to the reference (Minolta CM2600D) spectrophotometer measurement of a widely used Gretag Makbeth ColorChecker target.

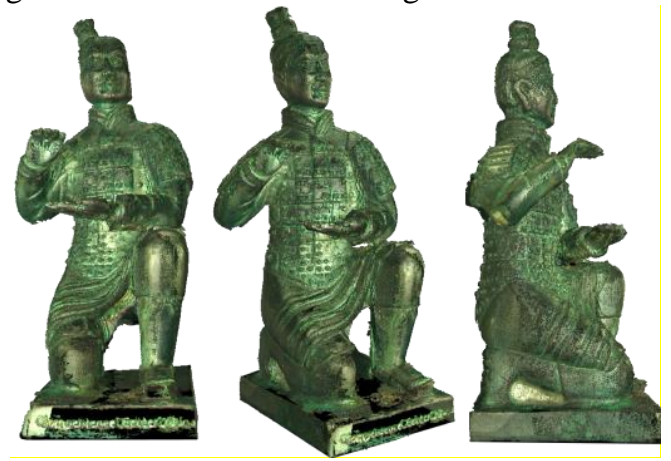

Fig. 7. Final result of digitization
In conclusion, we presented an integrated digitization method capable of capturing the shape, spectral and directional reflectance of an object. The built measurement setup is dedicated especially to documenting of cultural heritage objects, as it allows for both: capturing accurate surface characteristic with high precision and provides software tools for data processing and visualization. The ongoing research conducted in this field focuses on providing better solutions for merging data captured from different directions. It will benefit from known measurement system geometry so that energetic differences between reflectance registered from different directions can be eliminated in the early stage of data processing. Our aim is to provide a complete environment for art restorers, serving as digital documentation, helping to manage museum collections.

\section{References}

[1] J. Portal, Q. Duan, The First Emperor: China's Terracotta Army (British Museum Press, 2007)

[2] N. Yastikli, Journal of Cultural Heritage 8(4), 423 (2007).

[3] J. Salvi, Pattern Recognition 37(4), 827 (2004).

[4] S. Tosovic, R. Sablatnig, M. Kampel, Proc. of 7th Computer Vision Winter Workshop 2, 108 (2002).

[5] R. Sitnik, M. Kujawinska, J. Woznicki: Opt. Eng. 41, 443 (2002).

[6] F. Chen, G.M. Brown, M. Song, Opt. Eng. 39(1), 10 (2000).

[7] W. Mocko, Przeglad Elektrotechniczny 83(1), 39 (2007).

[8] E.A. Marszalec, M. Pietikaeinen, Proc. SPIE 3101, 170 (1997).

[9] A. Mansouri, A. Lathuilière, F.S. Marzani, Y. Voisin, P. Gouton, Multimedia IEEE 14(1), 40 (2007).

[10] F.H. Imai, M.R. Rosen, R. S. Berns, Proc. Eighth Color Imaging Conference, 234 (2000).

[11] F.H. Imai, M.R. Rosen, R.S. Berns, PICS conference, IS\&T, 185 (2001)

[12] G. Mączkowski, R. Sitnik, J. Krzesłowski, J. Im. Sci. Tech. 55(3), 030502-(10) (2011).

[13] F.E. Nicodemus, Appl. Opt. 4(7), 767 (1965).

[14] J.F. Blinn, ACM SIGGRAPH Computer Graphics 11(2), 192 (1977).

[15] H. Zhang, Z. Wu, Y. Cao, G. Zhang, G. Opt. Appl. 40(1), 197 (2010).

[16] S.R. Marschner, S.H. Westin, E.P. Lafortune, K.E. Torrance, Appl. Opt. 39(16), 2592 (2000).

[17] J. Krzesłowski, R. Sitnik, G. Mączkowski, Appl. Opt. 50(4), 532-541 (2011)

[18] B. Phong, Comm. of the ACM 18, 311 (1975).

[19] R. Sitnik, M. Karaszewski, Digital Heritage, 28-41 (2010).

[20] G. Wyszecki, W.S. Stiles, Color Science: Concepts and Methods, Quantitative Data and Formulae (John Wiley \& Sons, New York 2000).

[21] R. Sitnik, J. Krzesłowski, G. Mączkowski, Opt. Eng. 51, 021115 , (2012).

[22] R. Sitnik, M. Kujawińska, Proc. SPIE 5146, 54 (2003).

[23] P.J. Besl, N.D. McKay, IEEE Trans. Pattern Analys. Machine Intellig. 14(2), 239 (1992).

[24] R. Sitnik, G. Mączkowski, J. Krzesłowski, Digital Heritage, 13-27 (2010).

[25] R. Sitnik, J. Krzesłowski, G. Mączkowski, Internat. J. Heritage in Dig. Era 1, 25 (2012).

[26] IEC 61966-2-1:1999: Multimedia systems and equipment. Colour measurement and management. Colour management. Default RGB colour space. $s R G B$ 